Huntington's Chorea : Its Incidence in the Scottish Highlands. (Caled. Med. Journ., vol. xvi, p. 31, April, 1937.) McWilliam, $W$.

The writer found cases of Huntington's chorea confined to one fishing village on the east coast of Ross. All have the surname of either Patience or MacLeman, and appear to be descendants of fishermen who have drifted up north from the district round the Wash. It is interesting that American authorities trace most of 962 choreics back to four families who appear to have come from the east coast of England round the towns of Boston and Stamford. G. W. T. H. Fleming.

Prolonged Fever Following the Removal of Large Tumours from the Posterior Cranial Fossa. (Bull. Neur. Inst. New York, vol. vi, p. 33, Jan., 1937.) Ehrlich, W.

The author reports six cases of fever following the removal of a large glioma in the cerebellum, which lay in some cases in the midline, and in others in the hemispheres. In all cases the removal was accomplished by electro-surgical methods, and in all the wound healed without complication. The fever began within one to four days of the operation, reaching $103^{\circ}$ or $104^{\circ}$ in the evening, and falling to $99^{\circ}$ or $101^{\circ}$ in the morning. It persisted for more than five weeks in all patients, gradually falling throughout that period to normal. Four of the six patients appeared to be unaffected by the fever, and two complained of headache and other discomforts. No satisfactory explanation for the fever could be found in spite of repeated physical examinations, blood-cell counts, blood-culture and examination of the cerebro-spinal fluid. All the patients recovered. It is suggested that the fever might result from some profound disturbance in the circulation of the cerebro-spinal fluid.

T. E. BurRows.

\title{
5. Pharmacology and Treatment.
}

The Roentgen Treatment of Tumours of the Brain in the Operating Room by Direct Radiation through the Open Wound. (Bull. Neur. Inst., New York, vol. vi, p. 19, Jan., 1937.) Elsberg, C. A., Davidoff, L. M., and Dyke, C. G.

Following experimental roentgen radiation of the brain through the open wound in 16 Macacus rhesus monkeys, in order to determine the dosage, the method was applied to 18 patients with intracranial tumour. There was no evidence that there were any harmful effects as a result of the roentgen therapy. T. E. BuRRows.

Pharmacological Action of Three Derivatives of Choline. (Compt. rend. Soc. Biol., vol. cxxiv, pp. 276-9, 1937.) Wispelaere, M. de.

Acetyl- $\beta$-methylcholine (I), $\beta$-methylcholine ethyl ether (II), and $\beta$-ethylcholine (III) have a hypotensive action, respectively 20, 2 and Io times that of acetylcholine (IV). The heart-slowing action of I, and to a lesser extent that of II and III, is much more prolonged than that of IV. Atropine suppresses or inverts the hypotensive action of II and III and suppresses the action of small, but not large, doses of I. All 3 are intense excitants of the reflexogenic chemosensitive receptors of the carotid sinus.

(Chem. Abstr.).

General Paresis Treated by Mosquito-inoculated Vivax (Tertian) Malaria. (Amer. Journ. Psychiat., vol. cxiii, p. 619, Nov., 1936.) Kusch, E., Milam, D. F., and Stratman-Thomas, $W$. K.

The writers studied a group of 72 cases of general paresis treated with mosquitoinoculated vivax malaria, and compared the results with those of a former group of 363 cases treated with blood-inoculated malaria. They found that the course of vivax malaria is milder and better borne when induced through mosquito bite than when induced by blood inoculation. 